\title{
Expression of Toll-like receptor 4 on mast cells in gingival tissues of human chronic periodontitis
}

\author{
BO HUANG $^{1 *}$, QIAN DAI ${ }^{2 *}$ and SHI-GUANG HUANG ${ }^{2}$ \\ ${ }^{1}$ Science and Technology Park, Guangzhou University of Chinese Medicine, Guangzhou, Guangdong 510006; \\ ${ }^{2}$ School of Stomatology, Jinan University, Guangzhou, Guangdong 510632, P.R. China
}

Received June 21, 2016; Accepted April 27, 2017

DOI: $10.3892 / \mathrm{mmr} .2018 .8648$

\begin{abstract}
The purpose of the present study was to examine the expression of Toll-like receptor 4 (TLR4) on mast cells in gingival tissues of human chronic periodontitis. A total of 68 donors, including 23 with mild chronic periodontitis, 25 with advanced chronic periodontitis and 20 healthy controls, were included in the present study. Gingival specimens from the donors were fixed in $4 \%$ neutral formalin, stained with hematoxylin and eosin for histologic observation, stained for immunohistochemical identification of TLR4 in gingival tissues, and stained with double immunofluorescence for the identification of TLR4 on mast cells in gingival tissues. The results revealed that the expression of TLR4 in the gingival tissues and on mast cells in the gingival tissues of patients with chronic periodontitis were significantly higher, compared with those of the normal control group $(\mathrm{P}<0.05)$. The expression levels of TLR4 in the gingival tissues and on mast cells in patients with advanced chronic periodontitis were significantly higher, compared with those in patients with mild chronic periodontitis $(\mathrm{P}<0.05)$. In conclusion, the expression of TLR4 in gingival tissues and on mast cells increased with the severity of chronic periodontitis, suggesting that TLR4, particularly mast cell TLR4, may be important in the disease process of human chronic periodontitis.
\end{abstract}

\section{Introduction}

Mast cells have been generally considered to be specifically associated with allergic reactions. Increasing functions of mast cells have been identified, which indicate their relevance as effector cells and regulatory cells in innate and adaptive immunity $(1,2)$. However, the role of mast cells in the

Correspondence to: Dr Shi-Guang Huang, School of Stomatology, Jinan University, 601 Huangpu Avenue West, Guangzhou, Guangdong 510632, P.R. China

E-mail: huangshiguang_1@163.com

*Contributed equally

Key words: periodontitis, mast cell, Toll-like receptor 4 immunopathogenesis of periodontitis remains to be elucidated. Toll-like receptors (TLRs) are a type of recognition receptor with pathogen-associated molecule patterns, and are important in innate immune responses (3). As an important member of the TLR family, TLR4 is a receptor found in the lipopolysaccharide of cell walls in Gram-negative bacteria. Our previous study revealed that the presence of mast cells and degranulated mast cells were significantly increased in human chronic periodontitis tissues, indicating that the recruitment and degranulation of mast cells appeared to be associated with human periodontal disease $(4,5)$. However, at present, the association between TLRs and periodontitis remain to be fully elucidated. In the present study, toluidine blue and immunohistochemical stainings were used to identify mast cells. However, double-immunofluorescence was used for the identification of tryptase-TLR4 double-positive mast cells in gingival tissues. The aim of the present study was to examine the expression of TLR4 on mast cells in the gingival tissues of patients with human chronic periodontitis, to analyze the correlation between the expression of TLR4 on mast cells and periodontitis severity, and to investigate the potential roles of mast cell TLR4 in periodontitis using double immunofluorescence staining.

\section{Materials and methods}

Study subjects and periodontitis case selection. In the present study, subjects aged between 25-65 years who visited the Dental Clinic of the Affiliated Liwan Stomatological Hospital of Jinan University (Guangzhou, China) were recruited. The enrolled subjects consisted of 37 men (47 \pm 11.7 years old) and 31 women $(43 \pm 12.1$ years old $)$ and there were no significant differences in gender or age between each exprimental group. All subjects were free of diabetes or other systemic diseases; had not received any antibiotic treatment within the last 6 months, had no known drug or food allergy history, had not been pregnant or received any contraception therapy, and had not received periodontal surgery within the last year. The inclusion criteria were based on the American Academy of Periodontology (4) guidelines for the classification of periodontal disease and conditions in 1999 (6). All subjects signed informed consent. The present study was approved by the ethics committee of the Medical School of Jinan University. 
Grouping. The patients were divided into three groups, as follows: i) Healthy control group $(n=20)$, comprising individuals with no periodontal pockets, no periodontal attachment loss, no alveolar bone absorption on X-ray examination, no gingival inflammation; ii) mild periodontitis group, comprising patients with moderate periodontitis, gingival inflammation, bleeding on probing, pocket depths $\leq 4 \mathrm{~mm}$, attachment loss $\leq 2 \mathrm{~mm}$ and alveolar bone loss of no more than one third of the root length on X-ray examination, with or without halitosis; iii) severe periodontal group, comprising patients diagnosed with severe periodontitis presented with pocket depths $>6 \mathrm{~mm}$, attachment loss $\geq 5 \mathrm{~mm}$, alveolar bone loss of more than half or even two thirds of the root length on X-ray examination, furcation involvement on multiple teeth, loose teeth, periodontitis and possibly periodontal abscess presence.

Tissue specimens. The 23 tissue specimens from the mild periodontitis group, were obtained from those who required crown lengthening surgery; the 25 specimens in the severe periodontitis group were obtained from teeth with no reservation value, or with poor prognosis; the 20 clinically healthy specimens were harvested from the membranes of premolars extracted for orthodontic reasons.

Tissue specimen collection, processing and observation. The gingival biopsy specimens were fixed in $4 \%$ neutral buffered formalin for a minimum of $48 \mathrm{~h}$ prior to being cut into $5-\mu \mathrm{m}$-thick buccolingual serial sections, stained with hematoxylin and eosin, and observed under an optical microscope. The observation and grading of gingival inflammation was perfored by two pathologists in a blinded-manner. The average grade was then determined. The grade of inflammatory severity of the periodontal tissues was based on the scale provided by Huang et al (7) as follows: 0, no inflammation; 1, mild inflammation comprising focal inflammatory cell infiltrate; 2 , moderate inflammation comprising focal or patchy inflammatory cell infiltrate; 3 , severe inflammation comprising diffusive infiltration of inflammatory cells in gingival tissues.

SP immunohistochemical staining of TLR4 and processing. The SP immunohistochemical staining was performed to stain the gingival specimen sections. The dilution of I-anti-TLR4 antibody (cat. no. BA1717, Wuhan Boster Biological Technology, Ltd., Wuhan, China) was 1:100. The sections were stained using the SP immunohistochemical staining test kit (cat. no. PV9002; OriGene Technologies, Beijing, China), applied the DAB (DAB staining kit; OriGene Technologies) as the chromogenic reagents and substituted the I-antibody with PBS as the negative control. The specimens were then counterstained with hematoxylin. In the results of the histochemical staining, a positive signal was shown as small, brown granules, located in the nucleus or the cytoplasm. The number of positive cells was counted in each section. The total number of cells and number of positive cells in each of the tissue sections were counted in five representative visual fields (magnification, $\mathrm{x} 400$ ) and the positive ratio (\%) of the cells was determined. The positive ratio was the average percentage of postive cells in the total cells.

SP immunohistochemical staining of mast cells and TLR4, and processing. Double immunofluorescence staining was performed to stain the gingival specimen sections. The dilution of the I anti-tryptase antibody (cat. no. ab134932, Abcam, Cambridge, MA, USA) was 1:200. The dilution of the I anti-TLR4 antibody (cat. no. TA309486, OriGene Technologies) was 1:100. The dilution of goat anti-mouse IgG (H+L) Alex Flour ${ }^{\circledR} 555$ (cat. no. 4409; Cell Signaling Technology, Inc., Dallas, TX, USA), goat anti rabbit IgG $(\mathrm{H}+\mathrm{L})$ Alex Flour ${ }^{\circledR} 488$ (cat. no. 4412; Cell Signaling Technology, Inc.) was 1:200. Immunofluorescence positive signals showed as green fluorescence on mast cells and red fluorescence on TLR4, which were located in the nucleus or cytoplasm. When TLR4 and mast cells were superimposed in a single image, staining appeared yellow. Two pathologists observed the sections under an immunofluorescence microscope. They counted the numbers of TLR4-positive mast cells and calculated the average. The TLR4-positive mast cells were counted in five visual fields (magnification, $x 400$ ), which showed the highest yellow fluorescence.

Statistical analysis. All data are presented as the mean \pm standard deviation and were analyzed using SPSS 13.0 (SPSS, Inc., Chicago, IL, USA. Differences in gingival histopathological examination data between different groups were analyzed using Wilcoxon's signed rank test. The expression of TLR4 and the number of TLR4-positive mast cells in multiple groups were compared using completely randomized one-way analysis of variance. Multiple comparisons were compared using the least significant difference test. $\mathrm{P}<0.05$ was considered to indicate a statistically significant difference.

\section{Results}

Histopathological analysis. The histological results of the gingival tissues from different groups are shown in Fig. 1. No marked inflammatory infiltration was found in the healthy gingival tissues (Fig. 1A). The specimens in the mild periodontitis group showed moderate inflammatory cell infiltration (Fig. 1B). The speciments in the severe periodontitis group manifested with intense inflammatory cell infiltration, predominantly comprising lymphocytes, plasma cells and mast cells, and with a discrete presence of macrophage cells and polymorphonuclear cells (Fig. 1C). The severity of the grading of inflammation in the specimens from the different groups is shown in Fig. 2. The results indicated that the histological score of the periodontal tissues in the severe periodontitis group was significantly higher, compared with that in the moderate periodontitis group $(\mathrm{P}<0.05)$.

Gingival TLR4 immunohistochemical staining. The results of the gingival TLR4 immunohistochemical staining are shown in Fig. 3. The positive signal was observed as small brown granules, located in the nucleus or the cytoplasm. Only a few scattered TLR4 were observed in the healthy gingival tissues (Fig. 3A), whereas a higher number of TLR4-positive cells were observed in the mild periodontitis group (Fig. 3B) and the expression of TLR4 was even higher in the severe periodontitis group (Fig. 3C). The TLR4-positive cell ratios in the gingival specimens from the different groups are shown in Fig. 4. The results indicated that the expression of TLR4 in the gingival tissues of the chronic periodontitis group was 


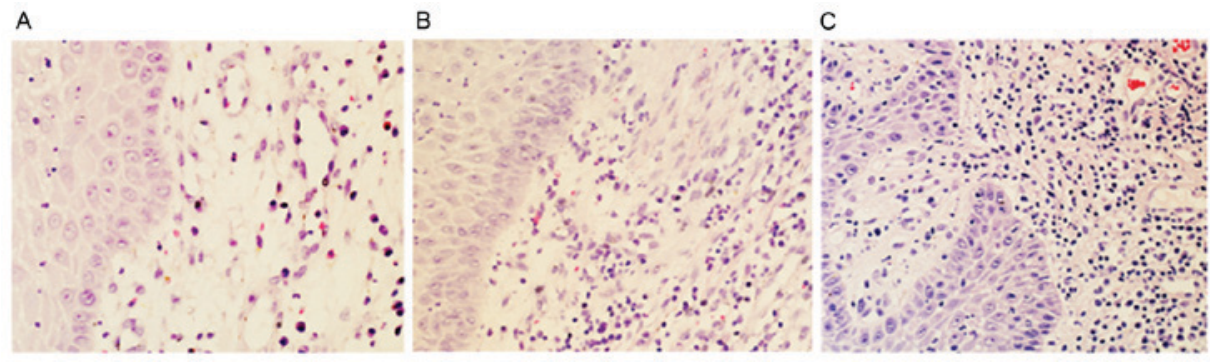

Figure 1. Representative photomicrographs of human gingival histology stained with hematoxylin and eosin (magnification, x400). (A) Healthy control; (B) mild chronic periodontitis group; (C) advanced chronic periodontitis group.

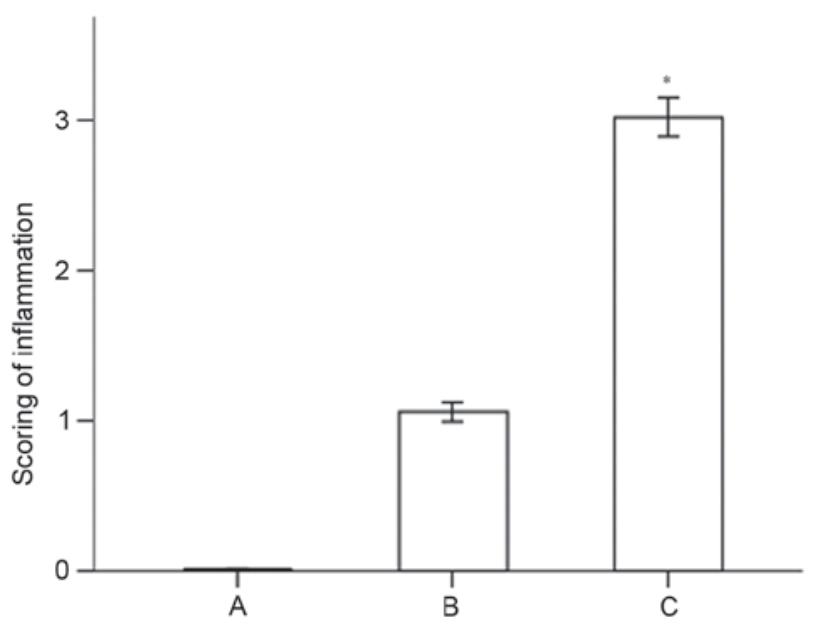

Figure 2. Severity of inflammation. Scoring of inflammation in the (A) healthy control $(n=20)$, (B) mild chronic periodontitis group $(n=23)$ and (C) advanced chronic periodontitis group $(n=25)$. Data are presented as the mean \pm standard deviation. ${ }^{*} \mathrm{P}<0.05$, vs. other groups.

significantly higher, compared with that of the normal control group $(\mathrm{P}<0.05)$. The expression of TLR4 in the gingival tissues of the advanced chronic periodontitis was significantly higher, compared with that of the mild chronic periodontitis group $(\mathrm{P}<0.05)$.

Immunohistochemical staining of mast cells and TLR4. Immunofluorescence-positive signals were shown as green fluorescence of mast cells and red fluorescence of TLR4, which was located in the nucleus or cytoplasm. When the TLR4 and mast cells were superimposed on each other, it appeared as yellow fluorescence. The immunohistochemical staining of the mast cells and TLR4 is shown in Fig. 5. A few scattered mast cells with TLR4 expression were observed in the healthy gingival tissues (Fig. 5A), whereas the number of mast cells and TLR4-positive mast cells in the gingival tissues was significantly increased in the mild periodontitis group (Fig. 5B). An increased number of mast cells and TLR4-positive mast cells were observed in the severe periodontitis gingival tissues (Fig. 5C). The numbers of TLR4-positive mast cells in the gingival specimens of the different groups are shown in Fig. 6. The expression of TLR4 on mast cells in the gingival tissues of the chronic periodontitis group was significantly higher, compared with that in the normal control $(\mathrm{P}<0.05)$. The number of TLR4-positive mast cells in the advanced chronic periodontitis group was significantly higher, compared with that in the mild chronic periodontitis group $(\mathrm{P}<0.05)$.

\section{Discussion}

TLRs are widespread biological pattern recognition receptors in mammalian cells. It can identify several conserved microbial surface structures, including bacterial lipopolysaccharides, peptidoglycan, lipoprotein, bacterial DNA and double-stranded RNA. By upregulating costimulatory molecule expression on the surface of antigen presenting cells (APCs), TLR signals, in addition to the inflammatory cytokines secreted by APCs, can induce $\mathrm{T}$ and $\mathrm{B}$ lymphocytes to differentiate into effector $\mathrm{T}$ and $\mathrm{B}$ lymphocytes, and adjust the adaptive immune response $(8,9)$. A previous study found that neutrophils, lymphocytes, macrophages and monocytes all exihibited TLR expression, and these TLRs were able identify different pathogens, rapidly activate innate immunity following pathogen invasion, trigger the inflammatory response and kill invading pathogens (10). As an important member of the TLR family, TLR4 is present in the lipopolysaccharide of Gram-negative bacteria walls (11). Periodontal diseases predominantly result from chronic pathological injuries, which are imposed on the periodontal tissues by gram-negative anaerobic bacteria, including Porphyromonas gingivalis. Studies have shown that the expression levels of TLR2, TLR3, TLR4 and TLR9 are significantly higher in tissues of patients with chronic periodontitis, compared with levels in the tissues of control individuals $(12,13)$. TLR2 polymorphisms were found to be closely associated with aggressive periodontitis in a Japanese population (14). TLR2 and TLR4 were also shown to be important in mediating osteoclast differentiation of alveolar bone in response to dyslipidemia (15). In periodontitis, a previous study found that the expression of TLR4 was identified predominantly in connective tissues, and that no TLR4 was expressed in the relatively healthy gingival tissues of patients with periodontitis or in healthy gingival tissues, indicating that TLR4 was involved in the periodontitis immune response (16). These results suggested that the expression level of TLR4 on human gingival fibroblasts reflects the extent of inflammation in the gingival tissues (17). Using immunohistochemistry, the present study found that the expression of TLR4 in the gingival tissue of patients with chronic periodontitis was significantly higher, compared with that in the tissue of the normal control group. The expression of TLR4 in the gingival tissues of patients with advanced chronic periodontitis was significantly higher, compared with that in tissues of patients 
A

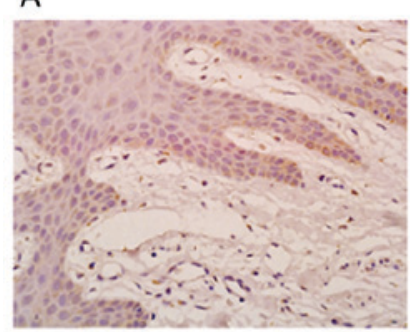

B

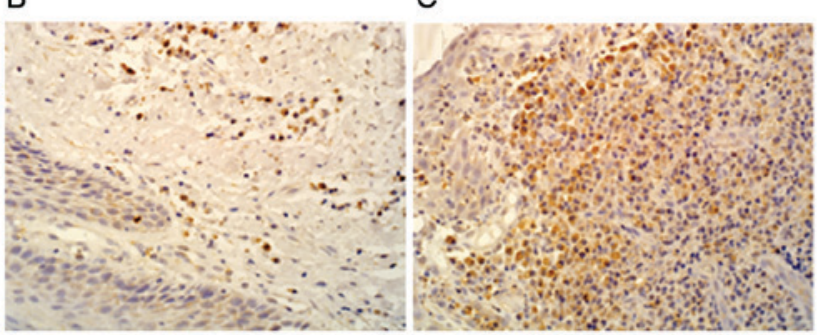

Figure 3. Expression of TLR4 in human gingival tissues. Immunohistochemical staining was performed to determine expression of TLR4 (magnification, x400). (A) Healthy control; (B) mild chronic periodontitis group; (C) advanced chronic periodontitis group. TLR4, Toll-like receptor 4.

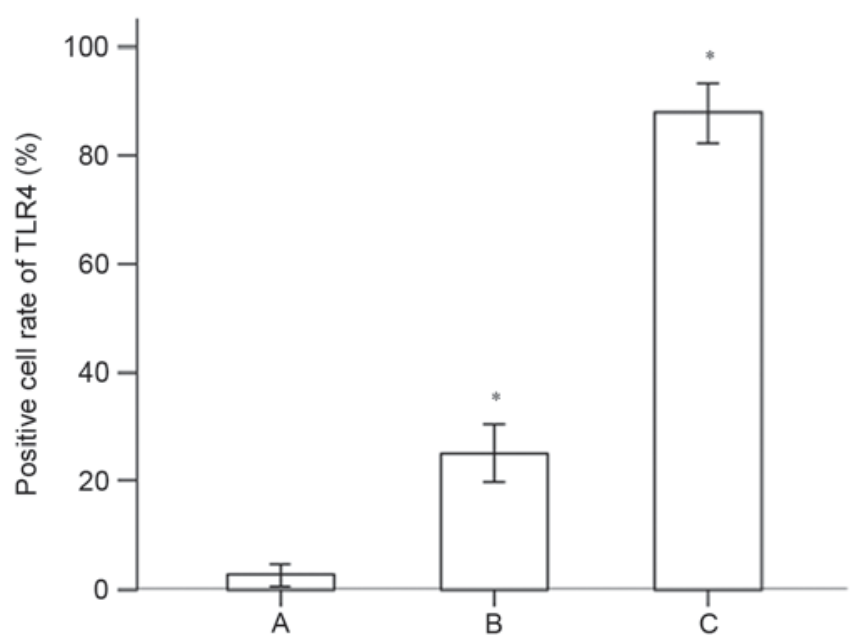

Figure 4. Expression of TLR. Positive cell rates of TLR4 in the (A) healthy control $(n=20)$, (B) mild chronic periodontitis group $(n=23)$ and $(C)$ advanced chronic periodontitis group $(\mathrm{n}=25)$. Data are presented as the mean \pm standard deviation. ${ }^{*} \mathrm{P}<0.05$, vs. other groups. TLR4, Toll-like receptor 4.

A
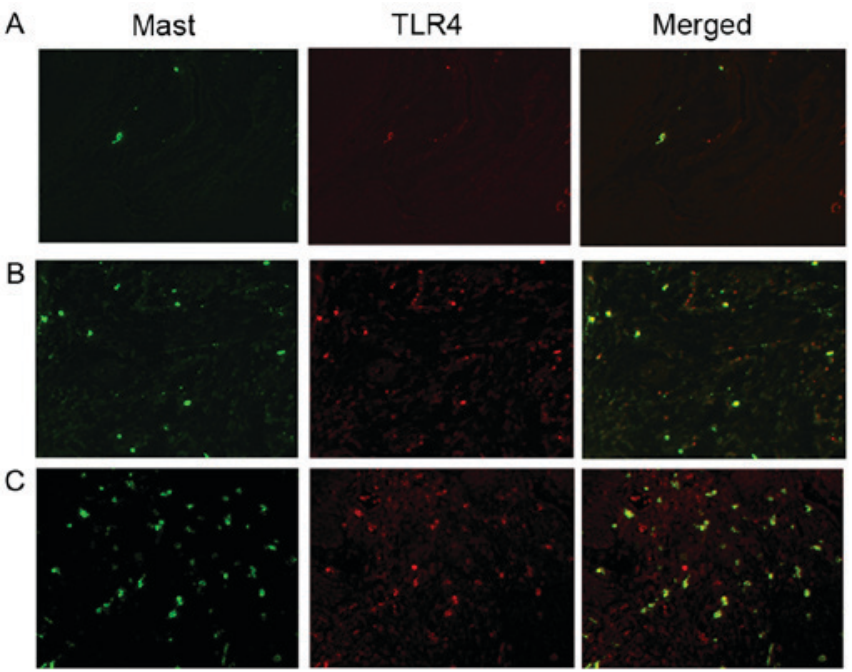

Figure 5. Expression of TLR4 on mast cells in human gingival tissues. Immunofluorescent staining was performed (magnification, $x 400$ ). (A) Healthy control; (B) mild chronic periodontitis group; (C) advanced chronic periodontitis group. TLR4, Toll-like receptor 4.

with mild chronic periodontitis, indicating a close association betwen the expression of TLR4 and the severity of chronic periodontitis.

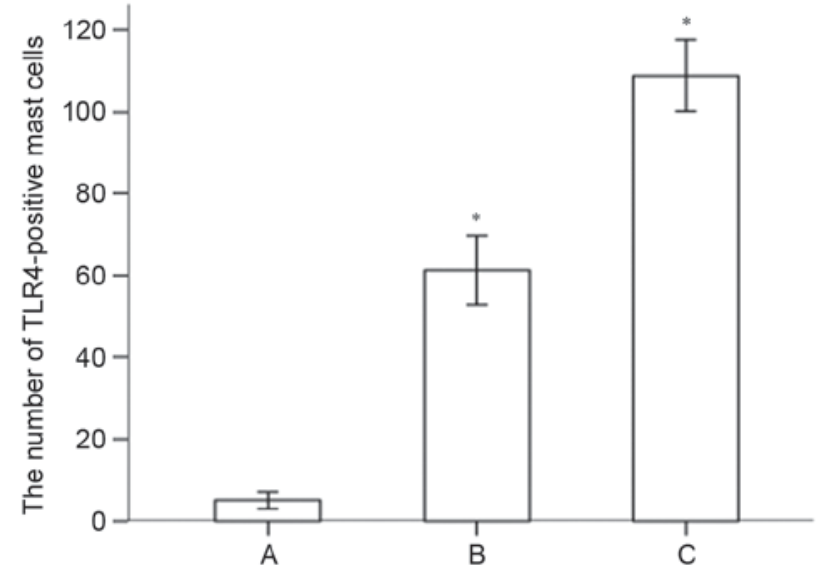

Figure 6. TLR4-positive mast cells. Total numbers of TLR4-positive mast cells in the healthy control $(n=20)$, mild chronic periodontitis group $(n=23)$ and advanced chronic periodontitis group $(n=25)$. Data are presented as the mean \pm standard deviation. ${ }^{*} \mathrm{P}<0.05$, vs. other groups. TLR4, Toll-like receptor 4 .

In previous years, the unique immune properties of mast cells have been increasingly acknowledged. In addition to the fact that mast cells are important in allergy and autoimmunity, mast cells are key effector and regulatory cells, and can initialize a rapid and continues immune response, which signifies their importance in innate and adaptive immunity $(18,19)$. The TLRs on the mast cell surface can identify a wide range of pathogen products and damage-associated molecular patterns (20). Mast cells activation can release pre-formed mediators such as histamine from their granules, as well as release de novo synthesized lipid mediators, cytokines, and chemokines. On receiving the corresponding stimulus, mast cells instantly release mediators, and initialize the innate immune response. Subsequently, they induce the inflammatory response or are involved in defence responses $(21,22)$. Bacterial components and inflammatory cytokines can regulate mast cell expression of TLR4. Following activation by TLRs, mast cells are not only engaged in the process of antibacterial defence and the inflamatory response, but they also affect the process of allergic reactions (23). A previous study found that activated TLRs promoted the generation of Th1-type cytokines and induced Th1 cell polarization (24). The TLR4 receptors on the surface of human and mouse mast cells can synergistically regulate the mast cell signaling pathway mediated by FcRI, and can promote mast cell degranulation and the release of Th2 cytokines (25). The present study showed that TLR4 
was expressed on mast cells in human gingival tissues from individuals with chronic periodontitis. The results showed that the expression of TLR4 on mast cells in the gingival tissues of chronic periodontitis was significantly higher, compared with that in mild chronic periodontitis, suggesting that mast cell TLR4 in human chronic periodontitis may be involved in the gingival inflamatiory reaction and is important in disease progression. However the mechanisms underlying how mast cells regulate periodontal disease via the identification of pathogenic bacterial antigens by TLR4 remain to be elucidated, and how mast cells regulate the onset and progression of periodontal disease through TLR4 signal transduction requires further investigation.

\section{Acknowledgements}

Not applicable.

\section{Funding}

This study was supported by National Natural Science Foundation of China (grant no. 81702020) and by Guangdong Provincial Science Foundation (grant no. 2015A030310107).

\section{Availability of data and materials}

The datasets used and/or analyzed during the current study are available from the corresponding author on reasonable request.

\section{Author contributions}

HS designed the present study and wrote the manuscript. HB and DQ performed experiments and analyzed data. All authors read and approved the final manuscript.

\section{Ethics approval and consent to participate}

All subjects signed informed consent. The present study was approved by the Ethics Committee of the Medical School of Jinan University.

\section{Consent for publication}

Not applicable.

\section{Competing interests}

The authors declare that they have no competing interests.

\section{References}

1. Shea-Donohue T, Stiltz J, Zhao A and Notari L: Mast cells. Curr Gastroenterol Rep 12: 349-357, 2010.

2. Zhang J, Alcaide P, Liu L, Sun J, He A, Luscinskas FW and Shi GP: Regulation of endothelial cell adhesion molecule expression by mast cells, macrophages, and neutrophils. PLoS One 6: e14525, 2011.

3. Mackern Oberti JP, Breser ML, Nuñez N, Maccioni M, Rodríguez N, Wantia N, Ertl T, Miethke T and Rivero VE: Chemokine response induced by Chlamydia trachomatis in prostate derived CD45+ and CD45- cells. Reproduction 142: 427-437, 2011.
4. Pan Q, LV FL, Huang B, Cheng Y, Liu M and Huang SG: Pathophysiological significance of mast cell degranulation in periodontitis. J Pract Stomatol 28: 316-319, 2012 (In Chinese).

5. Huang S, Lu F, Chen Y, Huang B and Liu M: Mast cell degranulation in human periodontitis. J Periodontol 84: 248-255, 2013.

6. Armitage GC: Development of a classification system for periodontal diseases and conditions. Ann Periodontol 4: 1-6, 1999.

7. Huang S, Lu F, Zhang Z, Yang X and Chen Y: The role of psychological stress-induced hypoxia-inducible factor-1 $\alpha$ in rat experimental periodontitis. J Periodontol 82: 934-941, 2011.

8. Iwasaki A and Medzhitov R: Toll-like receptor control of the adaptive immune responses. Nat Immunol 5: 987-995, 2004.

9. Liu J, Wu X, Chen ZC, Liao JD, Gu JY, et al: Comparison of Toll-like receptor-expressing cells from cord blood and adult peripheral blood granulocytes. Zhongguobinglishenglizazhi 29: 676-681, 2013 (In Chinese).

10. Hedayat M, Netea MG and Rezaei N: Targeting of Toll-like receptors: A decade of progress in combating infectious diseases. Lancet Infect Dis 11: 702-712, 2011.

11. Wei JH, Mo BW and Huang JW: Effect of TLR4/NF- $\kappa$ B on airway inflammation and airway remodeling in rat model of asthma. Chin J Pathophysiol 27: 962-967, 2011 (In Chinese).

12. Wara-aswapati N, Chayasadom A, Surarit R, Pitiphat W, Boch JA, Nagasawa T, Ishikawa I and Izumi Y: Induction of toll-like receptor expression by Porphyromonas gingivalis. J Periodontol 84: 1010-1018, 2013.

13. Buduneli N, Özçaka Ö and Nalbantsoy A: Salivary and plasma levels of Toll-like receptor 2 and Toll-like receptor 4 in chronic periodontitis. J Periodontol 82: 878-884, 2011.

14. Takahashi M, Chen Z, Watanabe K, Kobayashi H, Nakajima T, Kimura A and Izumi Y: Toll-like receptor 2 gene polymorphisms associated with aggressive periodontitis in Japanese. Open Dent J 5: 190-194, 2011.

15. Tomofuji T, Ekuni D, Azuma T, Irie K, Endo Y, Kasuyama K, Yoneda T and Morita M: Involvement of toll-like receptor 2 and 4 in association between dyslipidemia and osteoclast differentiation in apolipoprotein E deficient rat periodontium. Lipids Health Dis 12: 1, 2013.

16. De Oliveira NF, Andia DC, Planello AC, Pasetto S, Marques MR, Nociti FH Jr, Line SR and De Souza AP: TLR2 and TLR4 gene promoter methylation status during chronic periodontitis. J Clin Periodontol 38: 975-983, 2011.

17. Wang PL, Oido-Mori M, Fujii T, Kowashi Y, Kikuchi M, Suetsugu Y, Tanaka J, Azuma Y, Shinohara M and Ohura K: Heterogeneous expression of Toll-like receptor 4 and downregulation of Toll-like receptor 4 expression on human gingival fibroblasts by Porphyromonas gingivalis lipopolysaccharide. Biochem Biophys Res Commun 288: 863-867, 2001.

18. Bischoff SC and Krämer S: Human mast cells, bacteria, and intestinal immunity. Immunol Rev 217: 329-337, 2007.

19. Dietrich N, Rohde M, Geffers R, Kröger A, Hauser H, Weiss S and Gekara NO: Mast cells elicit proinflammatory but not type I interferon responses upon activation of TLRs by bacteria. Proc Natl Acad Sci USA 107: 8748-8753, 2010.

20. Dawicki W and Marshall JS: New and emerging roles for mast cells in host defense. Curr Opin Immunol 19: 31-38, 2007.

21. Tang $\mathrm{Y}$ and Huang T: Effects of mast cell degranulation on plaque stabilization in apolipoprotein E-Knock out mice. Chin J Arteriosclerosis 26: 1987-1988, 2010 (In Chinese).

22. Shelburne CP and Abraham SN: The mast cell in innate and adaptive immunity. Adv Exp Med Biol 716: 162-185, 2011.

23. Pietrzak A, Wierzbicki M, Wiktorska $M$ and Brzezińska-Błaszczyk E: Surface TLR2 and TLR4 expression on mature rat mast cells can be affected by some bacterial components and proinflammatory cytokines. Mediators Inflamm 2011: 427473, 2011.

24. Kaisho T and Akira S: Toll-like receptor function and signaling. J Allergy Clin Immunol 117: 979-988, 2006.

25. Novak N, Bieber T and Peng WM: The immunoglobulin E-Toll-like receptor network. Int Arch Allergy Immunol 151: 1-7, 2010. 